\title{
Jean Fernel, Felix Platter und die Begründung der modernen pathologischen Theorie
}

Von Antoinette Stettler

Krankheit und Krankheitsklassierung: Die Struktur der pathologischen Theorie

In der Medizingeschichte gilt im allgemeinen Giovanni Battista Morgagni als Begründer der Pathologie schlechthin, der in Antonio Benivieni im 15. und in Théophile Bonet im 17. Jahrhundert Vorgänger hatte und dessen Werk von Rudolf Virchow vollendet wurde. Die so gezeichnete Entwicklungslinie ist die Geschichte der Entdeckungen der pathologischen Befunde, die, immer weiter fortschreitend, die im Körperinnern sich abspielenden krankhaften Ereignisse sichtbar machten. Es ist dies die Geschichte der pathologischen Empirie.

Demgegenüber scheint die Geschichte der pathologischen Theorie nicht im Zentrum der Geschichtsbetrachtung zu stehen. Die pathologische Theorie entwickelte sich parallel zu den Entdeckungen und hat diese aufs Ganze gesehen nicht wenig im Sinne einer Wechselwirkung beeinflußt; neue Entdeckungen bewirkten Anpassungen der Theorie, und umgekehrt wurden oft auch die Entdeckungen durch den von der Theorie geschaffenen Rahmen bestimmt.

Sieht man von der Ansicht ab, daß Krankheit an sich gar kein medizinischer Begriff sei - und dafür spricht einiges -, so gehört die Frage nach der Krankheit selbstverständlich ins Gebiet der Pathologie. Diese «Krankheit» also tritt dem Arzt als Krankheitsbild, als Gestalt entgegen. Nicht etwa, daß er auf die Wahrnehmung der auf den naturwissenschaftlichen, physikalisch-chemischen Messungen beruhenden Fakten verzichten würde, aber er nimmt zunächst die Krankheit als Ganzheit in sich auf. Darnach wird er sein ärztliches Handeln ausrichten, das im besten Fall in der kausalen Therapie besteht.

Bei einigen Patienten ist nun eine solche Diagnose leicht zu stellen und die Krankheit unschwer zu heilen. Die Krankheit hat die Gestalt einer der uns bekannten Krankheitseinheiten und kann in das für uns gültige nosologische System eingeordnet werden. Eine kausale Therapie ist möglich. Eine solche Krankheit bietet weder ein pathologisch-nosologisches noch ein therapeutisches Problem. Das gilt namentlich für die Infektionskrankheiten (Chemotherapie), teilweise für die Tumoren (Chirurgie, Bestrahlung), für die Degenerationskrankheiten sowie für die Mißbildungen. Nebenbei sei bemerkt, daß die kausal zu behandelnden Krankheiten im großen ganzen den vier klassischen Virchowschen Kategorien der Pathologie entsprechen. 
Pathologisch-nosologische wie auch kausaltherapeutische Fragen stellen sich indessen heute in den medizinischen Spezialgebieten der Psychiatrie, der Dermatologie und der molekularbiologisch orientierten Medizindisziplinen wie z.B. der Hämatologie (sog. Hämoglobinanomalitäten, die keine klinischen Symptome zeigen, aber dennoch als Krankheiten bezeichnet werden). Diese drei Spezialgebiete haben dabei dieses gemeinsam: Das Kranksein spielt sich gewissermaßen an einer Trennwand von Innen und Außen ab. Ist die psychiatrische Krankheit als Fehlfunktion des ganzen Individuums mit seiner sozialen Außenwelt zu charakterisieren, so ist die dermatologische Krankheit eine Fehlfunktion der Haut als der äußeren Trennschicht des Individuums mit seiner körperlichen Umwelt. Die Rolle des Gegensatzpaares Außen/Innen würden im molekularbiologischen Bereich die Zellwand und das Plasma spielen, deren wie immer geartete Fehlfunktionen die Krankheit bedingen. Bis mindestens in die Zeit Virchows bildete dieses Gegensatzpaar, wörtlich verstanden, als Visualisierung die Zielsetzung der Pathologie.

Dem Gegensatzpaar Außen/Innen entspricht das Gegensatzpaar Schein/Sein. Für medizinische Belange ausgedrückt: was ist der Schein, das Anzeichen, das Symptom der Krankheit, und was ist das Sein, das «Wesenhafte» der Krankheit, gewissermaßen die Krankheit selbst? Sind Symptom und Krankheit erst einmal definiert, hat also aus den Mosaiksteinen der Symptome das Krankheitsbild - das Syndrom - Gestalt gewonnen, folgt, wenn man weiter zurückfragt, die Bestimmung der Ursache, was bekanntlich die schwierigste Aufgabe der Pathologie ist. Ursache oder Ursachen für die Krankheit gibt es unzählige. Die Meinungen schwankten von der multikausalen Ätiologie früherer Jahrhunderte zur monokausalen des 19. Jahrhunderts, der Triumphzeit der Bakteriologie, bis sie heute eher wieder der multikausalen zuneigen. Ist auch die Krankheitsursache bekannt, so kann eine der Dynamik der Krankheit gerecht werdende Pathogenese aufgestellt werden.

Ist die Relation des obengenannten Gegensatzpaares Symptom/Krankheit unbestimmt und die Krankheitsursache unbekannt, so stellen sich Fragen der pathologischen Theorie. Das Ziel jeglicher solcher Theorie ist selbstverständlich heute dasselbe wie zu allen Zeiten der Medizin und besteht eben darin, das Verhältnis der vier Pole Symptom, Krankheit, nosologische Verwandtschaft der Krankheiten sowie Krankheitsursachen zu bestimmen. In den konkreten Worten des handelnden Arztes ausgedrückt: hat diese bestimmte «abnorme Erscheinung» an diesem bestimmten Patienten als Symptom, als Krankheit, als Ursache zu gelten und muß sie als die gleiche Erscheinung oder als eine andere bei einem anderen Patienten angesehen werden? 
Ist Ziel und Vorgehen der Pathologie im Prinzip stets dasselbe geblieben, so war indessen die Struktur - im Sinne von Claude Lévi-Strau $\beta$ - der genannten vier Pole einem steten Wandel unterworfen und ist somit historisch. Der Strukturwandel der pathologischen Theorie kann so weit gegangen sein, daß Krankheiten desselben Namens (z.B. die Melancholie) nicht ohne weiteres miteinander verglichen werden können, wenn ihre Beschreibungen aus verschiedenen Epochen stammen, weil eben diese Beschreibungen von zu verschiedenartigen pathologisch-theoretischen Strukturen ausgehen. Ein Strukturwandel der pathologischen Theorie vollzog sich in der Renaissance, als sich im Werk von Jean Fernel (1506 bis 1558) die Modifizierung der seit der Antike beinahe unveränderten Theorie abzuzeichnen begann.

\section{Jean Fernels «Pathologia»}

Fernel gilt in der Medizingeschichte als nicht ganz erstrangiger Arzt des 16. Jahrhunderts. Die Sekundärliteratur beurteilt ihn in den letzten hundert Jahren entweder als letzte Vollendung des scholastisch-aristotelischen Mittelalters ${ }^{1}$ oder als echten Neuerer ${ }^{2}$. Fernel selbst verstand sich als Neuerer ${ }^{3}$, der die gesamte Medizin seinem Zeitalter anpassen wollte. In der Medizingeschichte wird er vom alles überstrahlenden Andreas Vesal in den Schatten gestellt. Doch hat Fernel in Paris im Jahre 1554 eine «Medicina» publiziert, deren Teil über die Pathologie noch 1751, also 10 Jahre vor der Publikation von Morgagnis «De sedibus ...», von Herman Boerhaave bzw. Albrecht von Haller als das bestgeeignete Lehrmittel seines Gebietes bezeichnet wurde ${ }^{4}$. Esmond Long ${ }^{5}$ nennt Fernel ohne ausführlicheren Nachweis den Begründer der Pathologie, so daß sich tatsächlich die Frage stellt, ob ihm nicht ein Platz neben Vesal gebühre.

Merkwürdigerweise befaßt sich der größte Teil der Sekundärliteratur, insbesondere das Standardwerk von Charles Sherrington, vornehmlich mit seiner «Physiologia », ${ }^{6}$ die auch einen Teil der «Medicina» bildet. Mary B. Hall ${ }^{7}$ hat nachgewiesen, daß diese «Physiologia» zu weiten Teilen mit Galens Buch «De naturalibus facultatibus» übereinstimmt, was Sherrington nicht aufgefallen sei. Das tut wiederum der Beurteilung Fernels als eines Neuerers Abbruch, weil man es bei dieser Auslegung bestenfalls mit einem Neugalenisten zu tun hätte. Tatsächlich herrschte zu Beginn des 16. Jahrhunderts in Paris eine Galenrenaissance, was sich leicht an den Galenpublikationen mit Gipfelpunkt um 1550 ablesen läßt ${ }^{8}$.

Gewiß ist Fernel, der in Paris zunächst Philosophie und Mathematik studierte, relativ spät Arzt wurde und als Hofarzt König Heinrichs II. starb, ein Neuinterpret Galens. Basiert die «Physiologia» auf Galens «De naturalibus facultatibus», so 
gründet seine «Pathologia», von der hier die Rede sein soll, auf Galens Büchern «De morborum differentiis», «De morborum causis», «De symptomatum differentiis», «De symptomatum causis» sowie «De locis affectis». Die Ähnlichkeit ist so groß, daß die Gedankenführung und auch die Kapitelfolge stellenweise genau übereinstimmen. Fernels Pathologia, Lib. I, Kap. 1-10, entspricht Galens De morborum differentiis; Pathologia, Lib. II, Kap. 4, entspricht Galens De symptomatum causis, Lib. I.; Pathologia, Lib. II, Kap. 5 und 6, ähneln Galens De symptomatum causis, Lib. II und III; Pathologia, Lib. II, Kap. 7-10, haben zahlreiche Beispiele aus Galen, De locis affectis, Lib. I.

Fernel daher als einen der im damaligen Paris recht zahlreichen Neugalenisten einzureihen, geht dennoch nicht an, wie der Blick auf diese Neugalenisten zeigt, welche die besagten Galenbücher entweder nur kommentierten ${ }^{9}$ oder in anderer Drucktechnik in Form von Tabellen ${ }^{10}$ publizierten. Vielmehr zeigt die Analyse der «Pathologia», daß noch andere Einflüsse als jene Galens bei ihrer Entstehung im Spiel gewesen sein müssen.

Fernel gehörte zum Kreise des Philosophen Pierre de la Ramée, der selbst kein revolutionärer Denker war, durch seinen Ideenreichtum indessen zum Ansporner für andere wurde ${ }^{11}$. In der Tat kann nachgewiesen werden, daß man sich im Paris der ersten Hälfte des 16. Jahrhunderts vermehrt mit Lukrez und dadurch mit den von Epikur überlieferten Vorsokratikern beschäftigte ${ }^{12}$. Man stellt dabei die Abwendung von der Naturlehre des Aristoteles fest, und zwar vor allem im Kreise um de la Ramée. Magnus Schmid ${ }^{13}$ macht darauf aufmerksam, daß für die Entstehung der «Physiologia» die Philosophie von Lukrez (De rerum natura), dadurch auch Epikur und möglicherweise die atomistischen Anschauungen Demokrits ausschlaggebend geworden sind. Die Auseinandersetzung mit den vorsokratischen Philosophen wurde, wie sich bei der Analyse der «Pathologia» zeigen wird, von größter Wichtigkeit.

Bevor wir uns nun der «Pathologia» selbst zuwenden, soll zunächst die Krankheitslehre, die Fernel zu Anfang des 16. Jahrhunderts vorfand, gekennzeichnet werden. Es handelt sich dabei um die genannten Galenbücher und um die «Mikrotechne» von Galen in arabischem Gewande. Letztere stand dem Arzt in Form der "Articella », ${ }^{14}$ erstere im pathologischen Teil des «Canon medicinae» des Avicenna zur Verfügung. Nicht daß die ursprünglichen galenischen Bücher dem Mittelalter gänzlich unbekannt gewesen wären. Es finden sich in den verschiedensten Bibliotheken noch einige Dutzend lateinischer Manuskripte. Doch aufs Ganze gesehen, rechtfertigt es sich, die galenische Pathologie in dieser arabisierten Form als typisch für die Zeit vor Fernel wiederzugeben, und zwar anhand der Ausdrucksweise des lateinischen Avicenna ${ }^{15}$ : 
Avicenna/Galen

Gegenstand der Krankheitslehre sind die widernatürlichen Zustände, die drei res contranaturales, nämlich:

- die Krankheit (affectus, aegritudo, morbus),

- die Ursache (causa),

- das Symptom (accidens, symptoma).

Krankheitsdefinition: « ... eine nicht natürliche Disposition im menschlichen Körper, aus der als Wesentliches ein Schaden der Körperfunktion hervorgeht. Sie ist entweder ein nicht natürliches Temperament [Dyskrasie] oder eine nicht natürliche Konfiguration. » ${ }^{16}$

Krankheitsklassen:

I. Einfache Krankheiten (aegritudo simplex) [sog. «innere» Krankheiten]

1. Temperamentskrankheiten (aegritudo complexionis) [Nach den Regeln der Kombinatorik können die vier Temperamente auf 16 Arten gestört sein, weshalb es 16 Arten von «intemperies» = Dyskrasie gibt.]

2. Konfigurationskrankheiten (aegritudo compositionis) [Organkrankheiten und Mißbildungen. Die Konfiguration der Organe kann nach Größe, Lage, Anzahl usw. verändert sein, was wiederum zahlreiche Unterklassen ergibt.]

3. Krankheiten der durchtrennten Körperkontinuität (aegritudo solutionis continuitatis) [Wunden, Brüche]

II. Die zusammengesetzten Krankheiten (aegritudo composita) [Diese sind aus den drei vorgenannten Krankheiten bzw. Unterkrankheiten zusammengesetzt. Als Beispiel: Apostema, tumor praeter naturam, was sowohl Temperaments- als auch Konfigurationskrankheit sein kann und oft Wunden aufweist.]

\section{Krankheitsursache:}

1. causa materialis [der kranke menschliche Körper]

2. causa formalis [die Temperamente]

3. causa finalis [die Körperfunktion]

4. causa efficiens [die Ursache im modernen Sinn: falsche Lebensweise, Körperdisposition sowie ein an sich vermeidbares krankmachendes «Etwas»]

Diese Ursachenlehre beruht auf Aristoteles, Physica, Buch II, Kap. 1-3.

\section{Symptom:}

«Symptom bedeutet Befall für den Betroffenen, aber Zeichen für den Arzt, der sich davon leiten läßt.» ${ }^{17}$ [Symptom/Zeichen gibt es anamnestische, demonstrative, prognostische; solche, die Art, Sitz und Ursache der Krankheit anzeigen, sowie wesentliche und zufällige.]

Zum Vergleich soll nun auch Fernels pathologische Theorie schematisch folgen, wobei betont sei, daß man damit seinem in sehr schönem Latein geschriebenen und sehr viel subtileren Text etwas $Z_{w a n g}$ antun muß. 


\section{Fernel:}

Gegenstand der Pathologia sind die drei Oberbegriffe (universalia):

- die Krankheit (morbus, affectus),

- die Ursache (causa efficiens),

- das Symptom (symptoma).

Krankheitsdefinition: " ... ein widernatürlicher Anfall, der im Körper innen sitzt.» ${ }^{18}$ "Was indessen eine widernatürliche Abweichung in den Körperinhalten [Blut, Säfte, Ausscheidungen, spiritūs vagi] ist, stellt die Krankheitsursache dar. Liegt aber der Fehler in den festen Körperteilen, hat man es mit der Krankheit selbst zu tun. Handelt es sich schließlich um eine Fehlfunktion, so steht man einem Symptom gegenüber. ${ }^{19}$

\section{Krankheitsklassen:}

I. Idiopathische Krankheiten

1. Gewebskrankheiten (morbus partis similaris) [Unterklassen: a. Immoderatio materiae; b. Intemperies = Dyskrasie; c. Corruptela totius substantiae $=$ Fieber, Vergiftungen, Pestilenzen.] ${ }^{20}$

2. Organkrankheiten (morbus instrumentarii) [Mißbildungen, Obstruktionen, Prolapse, Schielen usw. $]^{21}$

3. Krankheiten der durchtrennten Körperkontinuität ${ }^{22}$

4. Die zusammengesetzten Krankheiten (morbus compositus) ${ }^{23}$

II. Sympathische Krankheiten [sog. "Sekundärkrankheiten»] ${ }^{24}$

«Die Krankheitsordnungen sind ... nicht dem Wesen der Krankheit entnommen, sondern der Unterschiedlichkeit der festen Körperteile. ${ }^{25}$

\section{Krankheitsursache}

Nur causa efficiens

Die causae efficientes sind paarweise gruppiert:

externa/interna

intrinseca/adventitia [Gewichtigkeit der Ursache]

antecedens/continens [Entwicklungsweise der Ursache]

evidens/occulta

per se/per accidentem [Wirkungsweise der Ursache]

principalis/adiuvans/sine qua non [Quantifizierung der Ursache] ${ }^{26}$

Die causa adventitia externa evidens und die causa intrinseca antecedens und die causa intrinseca continens ergeben zusammen die «copulatio causarum» [Kausalitätskette]. Fernel legt damit dar, daß auf eine auslösende äußere Ursache hin ein Vorgang im Innern des Körpers beginnt, der dann seinerseits die Krankheit hervorruft, wobei er die causa beinahe als Kraft betrachtet, die diese Ursachenkette in Bewegung setzt und hält ${ }^{27}$.

\section{Symptom:}

I. die gestörte Funktion

1. die animale Funktion [Sensorik, Motorik, Verstand]

2. die natürliche Funktion 
II. das einfache, mit den fünf Sinnen wahrnehmbare Symptom

III. das Symptom in den Körperausscheidungen ${ }^{28}$

Krankheitsanzeichen (signum):

1. der Krankheit an sich

2. der Art und Ursache der Krankheit

3. das prognostische

4. das demonstrative ${ }^{29}$

Das System von Avicenna/Galen wie auch jenes von Fernel bilden eine vollständige und sachbezogene Krankheitslehre, die das oben skizzierte Ziel der Pathologie ansteuert. Doch haften beiden in sich abgerundeten Systemen gewisse Mängel an.

In erster Linie ist die Vorliebe für jeweils drei oder vier Kategorien zu nennen ${ }^{30}$. Diese Zahlenregelmäßigkeit hat zwar einen ausgesprochen ästhetischen Wert, birgt indessen die Gefahr in sich, die aus außermedizinischen Gründen erstrebte Regelmäßigkeit nun auch den medizinischen Belangen als angepaßt zu betrachten.

Zweitens sind beide Systeme der Temperamentenlehre verhaftet, die das Wissen über Wesen und Ursache der Krankheit als gegeben erachtete. Hier muß aber sogleich bemerkt werden, daß sich Fernel von der Temperamentenlehre schon so weit gelöst hat, daß er in seiner Krankheitsdefinition nichts von der Krankheit als Dyskrasie mehr sagt. Er läßt das Wesen der Krankheit charakteristischerweise offen: Die Krankheit ist im anatomischen Substrat zu suchen. Zwar ist die Idee des Ortes der Krankheit uralt und findet sich auch in Galens «De locis affectis », doch muß sie dort nicht so strikte wie bei Fernel auf die partes, die festen Körperteile, bezogen werden. Die Dyskrasie gibt es bei Fernel nur noch als eine der drei Unterklassen der Gewebskrankheiten.

Wenn drittens wie bei Avicenna/Galen die Begriffe Krankheit, Ursache, Symptom einander als res parallel gestellt sind, wenn sie also im System einander gleichgeordnet sind, ergibt sich leicht die Interpretation des Krankheitsgeschehens im Sinne einer Statik, die dem tatsächlich als dynamisch zu erklärenden Krankheitsablauf nicht gerecht werden kann. Demgegenüber betrachtet Fernel die drei «res» als Universalien im Sinne des Nominalismus, die er in eine Verbalverknüpfung setzt, was das Verständnis der dynamischen Krankheitsauffassung begünstigt: «Die Heilkunst handelt ... von der Krankheit und den Symptomen, von denen der Mensch befallen wird, sowie davon, was diese Symptome verursacht, und von den Zeichen, woran man sie erkennen kann. > $^{31}$

Viertens ist die Krankheitsklassierung der beiden Systeme etwas rudimentär und mit dem Konzept der zusammengesetzten Krankheit belastet, das ja der mo- 
dernen Medizin gänzlich fehlt. Wohl unterscheidet man mehrere Krankheiten bei einem und demselben Patienten, aber nicht eine aus mehreren zusammengesetzte Krankheit. Als Musterbeispiel der zusammengesetzten Krankheiten galten die Geschwülste und Schwellungen, die tumores praeter naturam. Von der Klassierung her ist dies eine durchaus verständliche Auffassung, und wir sehen heute leicht ein, daß das schwerfällige Konzept der zusammengesetzten Krankheit auf einer Mehrfachklassierung beruhte, was keinen Anlaß zur Aufstellung einer eigenen Klassierungskategorie, somit einer echten Krankheitsklasse, hätte abgeben dürfen. Die Klassierung war eben zu rudimentär, als daß sie eine sinnvolle und ordnungsgemäße Klassierung aller Krankheiten erlaubt hätte. So wurde zu solchen Mehrfachklassierungen Zuflucht genommen und die dadurch entstandene Krankheitsklasse irrtümlicherweise als echte Kategorie aufgefaßt. Auch Skrotalhernien, Phokomelien[Mehrfachklassierung in den Unterklassen der Organkrankheit] sowie auch fiebrige Pleuritis[Mehrfachklassierung bei den Dyskrasieunterklassen] usw. wurden für zusammengesetzte Krankheiten gehalten. Fernel wirkte nun insofern klärend, als er diesen Begriff ausschließlich für die tumores praeter naturam gelten ließ. Es blieb Felix Platter (1536-1614) vorbehalten, den Begriff der zusammengesetzten Krankheiten ganz aufzuheben und als der Klassierung inhärentes Problem zu durchschauen ${ }^{32}$. Das Konzept hielt sich trotz Platter zäh und ist erst mit Johannes Jonston (1603-1675) endgültig verschwunden, der wie Platter die Tumoren bei den Organkrankheiten einteilte ${ }^{33}$.

Fünftens ist darauf hinzuweisen, daß sowohl Avicenna/Galen wie auch Fernel trotz ihrer Krankheitsklassierung bei der Besprechung der einzelnen Krankheiten nicht nach dieser Klassierung, sondern nach der alten Methode De capite ad calcem vorgehen. Damit übernehmen sie die seit der Antike übliche Doppelspurigkeit der Klassierung, die in der für die Theorie bestimmten Krankheitsklassierung einerseits und andererseits in der auf die Praxis anzuwendenden De capite-Ordnung besteht. Damit ist gewissermaßen der Raster gegeben, der auch noch heute in der Trennung in eine allgemeine und spezielle Pathologie fortlebt. Man ist so sehr an diesen Raster gewöhnt, daß man ihn als eine der Medizin innewohnende Sache anzunehmen pflegt. Daß das aber nicht so zu sein braucht, wird das Beispiel Platters zeigen.

Ist bis jetzt von gewissen Mängeln der beiden Systeme die Rede gewesen, soll nun ihre Ähnlichkeit bzw. der Vorteil des Systems von Fernel beleuchtet werden. Oberflächlich betrachtet, ähneln sich die Systeme. Auf die Unterschiedlichkeit der Krankheitsdefinition haben wir schon hingewiesen; doch lassen sich auch bei der Krankheitsklassierung zwei recht wichtige Unterschiede zeigen, die dem System Fernels eine größere Flexibilität verleihen. Was Avicenna/Galen Tempera- 
mentskrankheiten nennen, sind bei Fernel drei Gewebskrankheiten, die der ebenfalls dreifachen Unterteilung der Gewebe in Fernels «Physiologia» entsprechen. Diese Dreiteilung stellt eine deutliche Erweiterung des medizinischen Gesichtsfeldes dar, indem der allzu umfassende Begriff der avicennisch/galenischen Dyskrasie gegliedert erscheint.

Wichtiger noch als die genannte Dreiteilung ist der Rückgriff auf Galen, den Fernel mit der Unterteilung der Krankheiten in idiopathische und sympathische macht. Idiopathische sind einem bestimmten Orte eigentümliche Krankheiten, sympathische solche, die an einen andern Ort im Körper vermittelt werden.

Dieses Begriffspaar erlaubt die gute Erfassung einiger Krankheitsbilder, wie z.B. die sympathische Lähmung der Extremitäten bei idiopathischer Rückenmarksverletzung oder die sympathische Blindheit bei Verletzung des Sehnervs oder das sympathische Kopfweh und Ohrensausen bei idiopathischer Magenverstimmung. Die sympathische Abmagerung bei verstopfter Vena cava caudalis ist indessen ein Beispiel eines falsch interpretierten Obduktionsbefundes ${ }^{34}$.

Das Begriffspaar Idiopathie/Sympathie dient nichts anderem als der Darlegung einer pathogenetischen Kausalverknüpfung, wie die Beispiele eindeutig zeigen. Fernel sagt, Sympathiekrankheiten seien eigentlich keine echten Krankheiten, es sei denn, es entständen daraus idiopathische, die dann als Sekundärkrankheiten und als von den ersten verursacht aufzufassen seien. In einem solchen Falle könne eine Krankheit gleichzeitig eine Ursache sein. Eine und dieselbe Erscheinung könne einmal in dieser, einmal in jener Kategorie der drei «res» eingeteilt werden.

Damit kommen wir zum Kernstück von Fernels «Pathologia», nämlich zu seiner Ursachenlehre, die sich durch die Aufhebung der aristotelischen Kausallehre ganz deutlich von Avicenna/Galen abhebt. Bezeichnenderweise berichtet Sherrington ${ }^{35}$, daß Fernel bei der Wahl des Titels zwischen «Aetiologia» und «Pathologia» geschwankt habe, ein Hinweis darauf, daß er die Ursachenlehre als ihren wichtigsten Teil ansah. Indem er sich nur mit der Causa efficiens beschäftigte, diese in der beschriebenen Art gruppierte und eine Kausalitätskette postulierte, kann er als Begründer der modernen Ätiologie gelten. Es ist zu vermuten, daß die Konzentration auf die Causa efficiens, mithin die Abwendung von der vielschichtigen aristotelischen Ursachenlehre, durch die vermehrte Beschäftigung mit vorsokratischem Gedankengut, vorab dem der Atomisten, gefördert wurde.

Nicht nur in den Kapiteln, die ausdrücklich von der Ursache handeln, befaßt sich Fernel mit dem ursächlichen Denken, sondern ganz allgemein ist sein Blick auf pathogenetische Zusammenhänge gerichtet, wie man schon bei den Idiopathie-/ Sympathiekrankheiten gesehen hat. Ein weiteres Beispiel für die Erhellung des 
pathogenetischen Zusammenhanges findet man in Fernels Überlegung zur Behauptung, die Krankheitsursache befinde sich in den Körperinhalten.

Dyskrasische Säfte können die Körperfunktion nur über den Umweg der Krankheit behindern; sie müssen die festen Körperteile erst durch Ansteckung (Einfluß von Fracastoros «De contagione ...», 1546?) angreifen. Fernel stützt diese Idee mit dem Hinweis auf die Malaria, die Tollwut und die Epilepsie, die alle drei ruhig liegende Ursachen in den Säften hätten ${ }^{36}$.

Auch seine auf den ersten Blick unnötig schwerfällig erscheinende Dreiteilung der Symptome dient dem Fahnden nach dem pathogenetischen Zusammenhang.

Das Hauptsymptom, die gestörte Funktion, ist z. B. die Obstruktion der Gallenblase, aus dem zwei Nebensymptome, das einfache, nämlich der Ikterus, und das Exkrementsymptom, nämlich der entfärbte Stuhl, folge ${ }^{37}$.

Abschließend ist noch Fernels Symptomlehre zu betrachten. Wie aufgefallen ist, hatte der Begriff symptoma/accidens im 16. Jahrhundert noch nicht die für uns eindeutige Bedeutung von Krankheitsanzeichen; vielmehr schwankte sie zwischen dem ursprünglichen Wortsinn «Befall, Anfall» - sich nicht prinzipiell von affectus (Krankheit) unterscheidend - und dem heutigen «Anzeichen». Avicenna/Galen gebrauchen das Wort je nachdem so oder so. Fernel fügt nun dazu noch das Wort signum, das zwar im Mittelalter auch gebräuchlich war; aber er macht einen grundsätzlichen Unterschied zwischen symptoma und signum.

Fernel bestimmt, daß das Symptom der Krankheit folge, sichtbar und außen am Körper sitze, wogegen das signum Zeichen einer sich tief im Innern befindenden Krankheit sei $^{38}$.

Auf diese Weise wird nun ein Unterschied zwischen symptoma und signum hergestellt, der nicht prinzipieller Art ist. Er wurde denn auch mit dem Häufigerwerden der Sektionen immer hinfälliger, weil bei ihnen Außen/Innen allmählich ineinander übergingen. Wenn aber Fernel dennoch diesen Unterschied machte, so deshalb, weil in seiner Zeit Autopsien noch keineswegs Routine, das Außen/ Innen noch durchaus unterschiedlich waren.

Aus der Schwierigkeit, daß symptoma begrifflich beinahe mit affectus (Krankheit) identisch war, hilft sich Fernel, indem er bestimmt, daß das Symptom der leichte, Krankheit der schwere Befall sei. Obwohl dieselbe Art von Befall, sei die Schwertwunde eine Krankheit, der Nadelstich ein Symptom ${ }^{39}$. Diese heute spitzfindig erscheinende Diskussion dreht sich um den Begriff der Normvariante.

In Analogie zu Avicenna/Galen ist auch für Fernel Symptom gleich Funktionsstörung. Fernel schlägt vor, die Gesamtheit der Symptome zu klassieren, und zwar solle man zwischen dem völligen Fehlen, der Beeinträchtigung und der Veränderung der Funktion unterscheiden. Am galenischen Beispiel des Auges bzw. des 
Ohres ergäbe das Blindheit, Schwachsichtigkeit und Halluzination (error et depravatio), bzw. Taubheit, Schwerhörigkeit und Ohrenläuten. Dieser Vorschlag fiel auf fruchtbaren Boden, indem Platter damit im ersten Band seiner «Praxis», 1602, mit dem Untertitel «De functionum laesionibus» Ernst machte und die erste moderne Klassierung erstellte.

Die eingangs aufgeworfene Frage, inwiefern Fernel als Neuerer oder Neugalenist zu bezeichnen sei, glauben wir dahin beantworten zu können, daß wir es entschieden mit einem Neuerer vom Range Vesals zu tun haben. Man stellt unschwer in der «Pathologia » die sprichwörtliche französische «clarté» und Eleganz fest; nur schon dadurch unterscheidet er sich von Galen und den Neugalenisten. Wohl ist er der spätscholastischen Philosophie wie auch den vorsokratischen Philosophen verpflichtet; doch schöpft niemand aus dem Leeren. Fernels entscheidende medizinische Leistung sehen wir darin, daß er das statische und zudem starr angewandte pathologisch-theoretische System des Avicenna/Galen mit der Dynamik der Pathogenese aufgebrochen hat, daß er das Kausalitätsdenken in die Medizin einführte und daß er schließlich die Krankheit im anatomischen Substrat suchte. Nicht erst Morgagni führte das anatomische Denken in die Pathologie ein ${ }^{40}$, sondern das tat bereits Fernel mit seiner Präokkupation vom Orte der Krankheit.

\section{Felix Platters «Praxis»}

Fernels Krankheitsklassierung war etwas zu rudimentär, als daß er auch darin als Begründer der pathologischen Theorie gelten könnte. Wohl hat er für die Strukturierung der beiden Pole Krankheit und Ursache Entscheidendes getan. Die Lehre der beiden anderen Pole, Symptom und Verwandtschaft der Krankheiten, (Nosologie im heutigen Wortsinne ${ }^{41}$ ), die zu einer kompletten pathologischen Theorie gehören, wurde von Platter modernisiert.

Die medizingeschichtliche Beurteilung Platters weist gewisse Ähnlichkeit mit derjenigen Fernels auf. Auch ihm, dem Basler Stadtarzt und Mitbegründer einer der gloriosesten Epochen der Basler medizinischen Fakultät, wagt man nicht die Stellung zu geben, die ihm gebührt. Sicher ist, daß er ein ausnehmend erfolgreicher Praktiker war, dessen Ruhm sich über die Grenzen seiner Vaterstadt bis gegen Polen hin erstreckte. Da zudem der größte Teil der Sekundärliteratur über Platter von Baslern stammt, könnte man meinen, daß hier aus einer Lokalgröße ein erstrangiger Arzt gemacht werden sollte. Das ist jedoch nicht der Fall, denn zur Zeit Platters war Basel alles andere als Provinz; man denke nur an die internationale Stellung des Basler Druckereigewerbes. In einer solchen Stadt hervorzuragen, ist an sich schon Hinweis auf eine erstrangige geistige Kapazität, und 
Platters Publikationen bestätigen diesen Rang. Als einer der ersten hat Platter Vesals Anatomie popularisiert und ein Memoirenwerk hinterlassen, das seinesgleichen sucht. Platter sollte in der Medizingeschichte etwa die gleiche Beurteilung erfahren wie Thomas Sydenham.

Auch Platter, der - eine Generation jünger als Fernel - in Montpellier studierte und auf der Heimreise nach Basel Fernel in Paris traf ${ }^{42}$, war noch insofern ein «Neugalenist», als er über Galens «De morborum causis» eine Probelektion in Basel hielt. Das zeigt, daß auch er dieselben Quellen zur Pathologie kannte wie Fernel. Platter war indessen kein Theoretiker im Sinne Fernels. Wenn wir ihn dennoch hier behandeln, so wegen seiner originellen Klassierungsleistungen in «De febribus» (1597) und in seiner «Praxis» (1602-1608) ${ }^{43}$.

Es ist schon auf die durch die Drucktechnik bevorzugte Darstellung einer komplizierten Materie in Tabellenform aufmerksam gemacht worden. Tabellen waren namentlich in der zweiten Hälfte des 16. Jahrhunderts eine in allen möglichen Gebieten beliebte Darstellungsart, die der Übersichtlichkeit und der Didaktik dienten. Besonders auch in Basel herrschte eine gewisse Tabellenfreudigkeit ${ }^{44}$. Dieser Methode bediente sich Platter in «De febribus». Neben der tabellenartigen Klassierungsform charakterisierte sich Platters Epoche auch durch Klassierungs- und Nomenklaturbestrebungen, namentlich in der Botanik (Conrad Geßner, Andrea Cesalpino, Caspar Bauhin). Auch Cesalpino habe übrigens den Vorschlag einer systematischen Klassierung der Krankheiten gemacht ${ }^{45}$. Platter selbst, der an botanischen Fragen interessiert war, erweiterte seine naturgeschichtliche Sammlung durch diejenige Geßners.

Allgemeine Tabellierungs- und Klassierungsbestrebungen sind auch für Platters «Praxis» wichtig geworden; für die Art und Weise seines Vorgehens ist indessen der Einfluß Fernels ausschlaggebend. Die «Praxis» erlebte etliche Auflagen und wurde 1662 ins Englische übersetzt. Die letzte lateinische Auflage erschien 1736, 31 Jahre vor dem Gipfelpunkt aller Nosologie, dem Monumentalwerk von François Boissier de Sauvages ${ }^{46}$.

Auch Platter kennt die drei Oberbegriffe der Pathologie. Doch er befreit sich davon und behält sich vor, ausschließlich von den Symptomen im Sinne Fernels $\mathrm{zu}$ handeln und seine «Praxis» darnach einzuteilen.

«Diese Zustände [Symptome] allein fallen unter die Sinneswahrnehmung, sind dem Kranken und seiner Umgebung augenfällig, führen ihn zum Arzt und werden ihm erzählt. ${ }^{47}$

Identifizierte Fernel das Wahrnehmbare und Konkrete mit der Veränderung am anatomischen Substrat (Innen gleich Krankheit), so war für Platter das 
Wahrnehmbare, das Außen, gleichbedeutend mit Symptom. Sein auf das mit den fünf Sinnen Wahrnehmbare gerichteter Geist läßt auch seine Aussage verstehen:

«... was unbekannt ist, will ich eher aus den Wirkungen als aus den Ursachen beurteilen. ${ }^{48}$

Für Platter ist die Ursache nur noch die Causa efficiens. Die Dyskrasie sei überhaupt keine Krankheit, sondern eine Ursache, dergestalt, daß z. B. die Ursache Dyskrasie des Magens oder der Leber die wahrnehmbaren Symptome Nausea, Erbrechen, Kopfweh, Kachexie und Hydropsie hervorrufen könne. Auch scheut sich Platter, etwas über das Wesen der Krankheit auszusagen. Seine Krankheitsdefinition ist funktionell:

«Unter den zahlreichen und beinahe unendlich vielen Krankheiten ..., die der Unversehrtheit und dem Leben des Menschen auflauern, bald die Tätigkeit behindern, schwächen und verschlechtern, bald ihn mit Schmerzen schlagen,... bald den Körper verunstalten und behindern, finden wir keine häufiger als die Fieber. ${ }^{49}$

Wenn wir behaupten, Platter scheue sich, etwas über das Wesen der Krankheit auszusagen, und messe dem Symptom alle Wichtigkeit bei, meinen wir damit nicht, er habe nur noch das Einzelsymptom gesehen. Das Gegenteil ist der Fall. Er betrachtete die Krankheit als Synthese der Einzelsymptome, die er als Krankheitsbild verstand. Er befreite damit den Arzt von der meist unfruchtbaren Diskussion über das «Wesen» der Krankheit und lenkte den Blick auf die wahrnehmbare Erscheinung, auf die Symptomatologie. So ist denn die Klassierung, die Platter in seiner «Praxis» vornahm, eine Klassierung nach Symptomen bzw. Syndromen und im strengen Sinne keine Krankheitsklassierung; denn die nach damaligem Gebrauche sogenannten Krankheiten treten bei Platter eben gerade nicht als Kategorien auf. Platters Syndromklassierung wurde von Albrecht Burckhardt in seiner ausgezeichneten Geschichte der Basler medizinischen Fakultät ${ }^{50}$ ausführlich behandelt, so daß wir auf ihre $\mathbb{W}$ iederholung verzichten. Immerhin muß zum Verständnis des Zusammenhanges kurz angedeutet werden, worum es sich handelt.

Wie in Platters Krankheitsdefinition vorweggenommen wurde, scheidet er seine «Praxis» in drei Teile: «De functionum laesionibus», «De doloribus» und «De vitiis». Die gestörten Funktionen sind die des inneren Sinnes und der Sinnesorgane, die nach dem Vorschlage Fernels in völliges Fehlen, Beeinträchtigung und Veränderung kategorisiert sind, sowie die Störungen der Motorik, die nach demselben Vorgehen klassiert werden. Die Schmerzen werden als Schmerzen « an sich », als Schmerzen bei Fieber, Rheuma, Gicht usw., drittens als Schmerzen der Körper- 
oberfläche unterteilt. Die «vitia », die Verunstaltungen, betreffen grobe organische Veränderungen des Körpers und der Exkretion, somit auch die tumores praeter naturam, das Musterbeispiel der ehemals zusammengesetzten Krankheiten. Wie jede Klassierung weist auch die Plattersche einige Schwächen auf (so, wenn er unter Störungen der Motorik die Lähmungen und Brüche zusammen einordnet, was schon Burckhardt als ungeschickt empfand). Doch erlaubten Platters Klassierungskriterien ein weitgehend natürliches Einteilen, so daß seine Einordnung der Störungen des inneren Sinnes, der Geisteskrankheiten also, auch heute ihre Berechtigung noch nicht ganz eingebüßt hat. Die antike Funktionenlehre, dreigeteilt in Animal-, Natural- und Vitalfunktionen, eine Psychophysiologie, wie man heute sagen würde, war eben derart vollständig, daß sie ein natürliches Klassieren ermöglichte.

Zum Schmerz als Klassierungskategorie soll folgendes beigefügt werden: Der Schmerz, eine wahrhaft alltägliche Erscheinung, wurde erst in der zweiten Hälfte des 16. Jahrhunderts Gegenstand besonderen Interesses. Es erschienen damals zahlreiche kürzere Monographien und Dissertationen zu diesem Thema ${ }^{51}$. Platter konnte hier nicht wie bei den Funktionen auf eine vollausgebildete Lehre zurückgreifen. In der arabischen Überlieferung galten Schmerzen bisweilen als Ursache von Krankheiten (Schmerz als Ursache des Furunkels ${ }^{52}$ ). Seit Fernel die Schmerzen zu den demonstrativen Krankheitszeichen zählte und Platter sie aus Gründen der Wahrnehmbarkeit zu einem seiner drei Klassierungskriterien erhob, wurde die oft beanstandete Entwicklung des Schmerzes als einem wichtigen Diagnosezeichen eingeleitet. Aus dieser Stellung wurde der Schmerz zu Beginn des 19. Jahrhunderts mit dem Bekanntwerden weiterer neurophysiologischer Einzelheiten wieder etwas abgedrängt.

Die Gruppe der Verunstaltungen umfaßt die Krankheiten mit morphologischen Veränderungen und die von Fernel als «signa» bezeichneten Exkrementsymptome. Das ist ein weiter Begriff, so daß darin viel nicht Homogenes Aufnahme gefunden hat. Dennoch sind die Partialklassierungen, die der Band «De vitiis» enthält, von der Sache her gerechtfertigt.

Platters Leistung zur pathologischen Theorie nahm im täglichen Umgang mit Patienten ihren Ursprung. Es ist der Beitrag des handelnden und reflektierenden Arztes. Deshalb ist für ihn das Symptom als das sinnlich Wahrnehmbare, das Außen, das Ausschlaggebende, dem er alles, namentlich die Diskussion um das Wesen der Krankheit, unterordnete. Seine einheitliche und umfassende Klassierung der Syndrome ist die erste Nosologie im modernen Sinne. Sie überwand zum Nutzen der Medizin die Doppelspurigkeit der Klassierung, wie sie noch Fernel gebrauchte. Es ist wohl kein Zufall, daß nach Platter zahlreiche ausgezeichnete Monographien 
über einzelne Krankheiten geschrieben wurden und daß gerade in England (siehe auch die englische Übersetzung der «Praxis») mit Richard Morton (1637-1698) so treffliche Partialklassierungen ${ }^{53}$ entstanden. Die Aufnahme des Werkes von Platter in England wäre ein interessanter Untersuchungsgegenstand.

Die Übersicht über das Werk Fernels und Platters zeigt, daß der eine in der Struktur der pathologischen Theorie die Begriffe Krankheit und Ursache, der andere die Begriffe Symptom und Klassierung klärte. Somit fand diese Struktur dem Umriß, den sie auch heute noch hat.

\section{Ausblick auf das 17. und 18. Jahrhundert}

Es soll keineswegs eine vollständige Rezeptionsgeschichte der pathologischen Theorie, wie sie von Fernel und Platter begründet wurde, folgen. Vielmehr wird nur den vier erwähnten strukturellen Polen Aufmerksamkeit geschenkt.

Im 17. Jahrhundert wurden die Autopsien, die im anatomischen Substrat die Krankheit suchten, so häufig, daß Bonet 1679 sein «Sepulchretum» veröffentlichen konnte, worin er u. a. auch Sektionsbefunde (Tuberkulose), die von Fernel gemacht worden waren, verwendete ${ }^{54}$.

Daniel Sennert (1572-1637), genannt «Galenus germanicus», ${ }^{55}$ darf als Vertreter der Ideen Fernels in Deutschland gelten. In Breslau geboren, war er Chemiker und Professor der Medizin in Wittenberg. Er hat in beiden Gebieten umfangreiche Werke hinterlassen. Sich selbst betrachtete er als einen Vermittler zwischen Galen und den neuen chemisch-therapeutischen Erkenntnissen, die auf Paracelsus zurückgehen. Nach seinem Studium soll sich Sennert bei Felix Platter in Basel aufgehalten haben ${ }^{56}$, was in das Jahr 1601 gefallen sein müßte. Doch verzeichnet die Universitätsmatrikel seinen Namen nicht.

Seine vorwiegend iatrochemischen Neigungen lassen zunächst den Einfluß Fernels auf sein pathologisches System, das sich in seinen Werken «Institutiones», «Praxis» und «Paralipomena ${ }^{57}$ findet, nicht vermuten, doch zeigt ein Vergleich beider Systeme, daß Sennert wohl eher ein «Fernelius germanicus» war. Er steht Fernel indessen nicht unkritisch gegenüber, ist in Einzelheiten auch manchmal, zumal in den früheren Werken, galenischer als Fernel; doch gleicht sein System im ganzen sehr demjenigen Fernels mit der Ausnahme, daß bei Sennert, dem Chemiker, die Unterteilung der Vergiftungen weiter fortgeschritten ist ${ }^{58}$. Fernel zählt die Vergiftungen zu den Krankheiten der ganzen Substanz, wogegen Sennert zum Namen «morbus occultae qualitatis» greift. War für Fernel das Innen das Verborgene, noch nicht durch Autopsie Wahrgenommene, so stellte es sich für Sennert dar als die noch nicht durchschaubaren chemischen Vorgänge der Vergiftung 
im Inneren des Körpers. Man darf daher seine Lehren als Weiterentwicklung von Fernels «Pathologia» im Sinne einer Annäherung an die Iatrochemie auffassen.

Auch Platters neuartige Klassierung war Sennert bekannt. Er lobt sie zwar sehr, hat sie aber nicht angewendet, möglicherweise, weil er sie zu spät erfahren hat, möglicherweise bewußt. Er äußert sich dazu folgendermaßen:

«Eine einzigartige und ungebräuchliche Methode hat der berühmte Felix Platter befolgt ... Was aber bei seiner Methode wirklich zu wünschen übrigläßt, ... ist, daß er oft verschiedene Krankheiten am selben Orte und dagegen solche, welche verwandt sind, an verschiedenen Orten behandelt..., so daß man, wenn man die Lektüre nicht fortlaufend pflegt und den Index nicht ständig vor Augen hat und konsultiert, viele Krankheiten nicht leicht findet. » ${ }^{59}$

Damit legt Sennert den Finger auf das Problem des Rasters, auf das wir im Zusammenhang mit der Doppelspurigkeit der Klassierung hingewiesen haben. Eine einzige Klassierung setzt einen guten Index voraus. Möglicherweise aber war für Sennert Platters Methode mit der Ausklammerung aller bisher gebräuchlichen «Krankheiten» zu revolutionär, als daß er ihren Wert eingesehen hätte.

Einen lehrreichen Überblick über den Synkretismus der pathologischen Theorie um die Mitte des 17. Jahrhunderts ergibt das Werk «Idea universae medicinae» von 1644 des Johannes Jonston, der Stadtarzt zu Lissa bei Posen und ein naturgeschichtlich berühmter Enzyklopädist war. Er versteht seine Schrift, ein Buch über Bücher, als "perspektivische Darstellung fast aller Krankheiten », ${ }^{60}$ womit er einen graphisch-architektonischen Ausdruck zur Charakterisierung seines «Systembaus» verwendet. In der Tat zeigt sich dann im 18. Jahrhundert eine Verwandtschaft der pathologisch-theoretischen Systeme mit der Architektur, auf die Philippe Pinel hinweisen wird ${ }^{61}$.

Die Stellung von Franz de le Bö̈ Sylvius (1614-1672) in der Medizingeschichte ist unbestritten. Nicht nur hat er sich um die Verbreitung von William Harveys Entdeckung des Blutkreislaufes verdient gemacht, sondern er hat auch durch eigene Forschungen an den Drüsen das anatomische und physiologische Wissen bereichert. Seine auf Jan Baptist van Helmont beruhende iatrochemische Fermentationslehre, wonach die normalen und pathologischen Lebensvorgänge auf chemischer Umsetzung beruhen, hat großes Aufsehen erregt. Auch Sylvius hat eine neue Krankheitsklassierung aufgestellt, die ebenso originell wie jene Platters ist und nichts vom Synkretismus Jonstons hat.

Sein Vorgehen bei der Klassierung bezeichnet er als «... der Methode Platters nicht unähnlich ». ${ }^{62}$ Auch Sylvius klassiert die Krankheiten anhand der Funktionsstörung. Die traditionelle Lehre der drei Funktionen erfährt indessen eine be- 
zeichnende Straffung. Die Teilfunktionen der drei Hauptfunktionen werden so umgruppiert, daß die Nahrungsaufnahme, das Wachstum, die Sinnesempfindung und die geistige Tätigkeit - lange vor der heutigen Psychologie - zur Selbsterhaltungsfunktion (functio individui conservando) zusammengefaßt werden. Davon abzutrennen sind die Fortpflanzungsfunktionen, die der Arterhaltung (speciei propagatio) dienen ${ }^{63}$. Die Lebenskraft, bisher eine Teilfunktion, hat in diesem System keinen Platz mehr, was selbstverständlich mit der von ihm geschaffenen iatrochemischen Pathogenese zu tun hat. Bezeichnenderweise handelt Sylvius auch nicht von den Hautkrankheiten und Tumoren, so daß sein System, das hauptsächlich die Verdauungskrankheiten schön plaziert, weniger vollständig ist als dasjenige Platters. Dessen Klassierung der Geisteskrankheiten wurde von Sylvius in den großen Zügen übernommen, wobei er aber weniger subtil als der gut beobachtende Platter vorging, was sich aus seinen iatrochemischen Interessen erklären mag. Damit beginnt sich wohl die Abtrennung der Psychiatrie von der Körpermedizin abzuzeichnen.

Ein eigentümlicher Gedanke für die Krankheitsklassierung ging von Thomas Sydenham (1624-1689) aus. Er befürwortete die Anwendung der botanischen Methode auf die Medizin, eine Idee, die er vielleicht in seiner Studienzeit in Montpellier aufgenommen hatte. Zwar hat auch schon Cesalpino die botanische Methode empfohlen, doch ging Sydenham einen Schritt weiter. Er findet, daß vor aller Therapie die Krankheiten besser beobachtet werden müßten; dabei soll der Arzt wie der Botaniker vorgehen, der eine Pflanzenart in allen ihren wesentlichen Einzelheiten beschreibt und nicht so ungenau ist, daß er bei der Gattung stehenbleibt ${ }^{64}$. Bekanntlich sind Sydenham mit dieser «botanischen» Methode außerordentlich gute Krankheitsbeschreibungen gelungen. Es ist schwer, bei Sydenham zu entscheiden, inwiefern er sich das Vorgehen als Analogie zur Botanik vorstellte und inwiefern er an die letztliche Existenz einer Krankheitsspezies glaubte.

Der Begriff der Krankheitsspezies wurde für François Boissier de Sauvages (1706-1767), Professor der Medizin in Montpellier, wegleitend, indem er die Klassierung der Krankheiten in botanischer Manier betrieb. Seine Einteilung enthält Klassen, Ordnungen, Gattungen und Arten, so daß z. B. der Klasse vitia die Ordnung maculae, dieser die Gattung leucoma, dieser wiederum die Spezies des lokal vorkommenden Leukoms untergeordnet sind ${ }^{65}$. Sein ausschließlich auf Nosologie gerichtetes Bestreben läßt sich großenteils aus dem ausgeprägten Hang des 18. Jahrhunderts zu systematischen Überblicken verstehen. Ein Großteil der Ärzte teilte diese Neigung und trat - beinahe wie mit einem Statussymbol - mit einer eigenen Nosologie an die Öffentlichkeit ${ }^{66}$. Boissier de Sauvages blickte be- 
reits methodebewußt und kritisch auf die Versuche Sennerts und Jonstons. Platters Nosologie scheint ihm dagegen nicht bekannt gewesen zu sein.

Wenn sich Boissier auch nicht als direkter Nachfolger Platters einstufen läßt, so ist das Ziel und Ergebnis seiner Unternehmung dennoch dasselbe, wie sich auch aus seiner Krankheitsdefinition ersehen läßt.

«Die Krankheiten sind das Zusammentreffen der auffälligen, wechselseitig verbundenen Symptome. Die mehr auf der Hand liegenden und gleichzeitig konstanteren Symptome führen den Zug [der Krankheitsbeschreibung] an und bestimmen den wesentlichen Charakter der Krankheit. » ${ }^{67}$

Krankheit ist Syndrom, Krankheitsbild geworden - auch species heißt Bild. Nosologie, die von Platter eingeleitete Forschungsrichtung, diente der exakten Beobachtung der Krankheitsgestalt. 1761, etwa gleichzeitig, bestimmte Morgagni, den Boissier bekämpfte, die Krankheit als anatomische Veränderung und untermauerte mit seinen zahllosen Belegen Fernels Idee vom Ort der Krankheit. Wenn dann im 19. Jahrhundert die reine Nosologie immer ungebräuchlicher, die pathologische Anatomie dank Virchow immer vorwaltender wurde, so hat diese Entwicklung zu einer Zweiteilung der Medizin beigetragen, die nicht mehr die Ganzheit des Krankseins im Zentrum des Blickfeldes hatte. Daß dies nicht so sein muß und nicht immer so war, zeigen uns die beiden Ärzte der medizinischen Renaissance, die wir hier behandelt haben.

Dieser Artikel ist die Kürzung eines 48seitigen Manuskriptes, das wegen der Druckkosten nicht publiziert werden konnte. Die Autorin gibt über weitere Belege, namentlich über Sennert, Jonston und Sylvius, gerne Auskünfte. Das Material zu dieser Arbeit wurde in großzügigster Weise von der National Library of Medicine, Bethesda/Maryland, USA, zur Verfügung gestellt. Herrn Prof. Dr. med. H. M. Koelbing, Universität Zürich, bin ich für viele wertvolle Hinweise zu großem Dank verpflichtet. 


\section{Anmerkungen}

1 A. Herpin, Jean Fernel, médecin et philosophe, Paris 1949.

K. E. Rothschuh, Das System der Physiologie von Jean Fernel (1542) und seine Wurzeln. In: Aktuelle Probleme aus der Geschichte der Medizin (Verhandlungen des XIX. Internationalen Kongresses für Geschichte der Medizin, Basel 1964), Basel/New York 1966, S. 529-536.

${ }^{2}$ L. Figard, Un médecin philosophe au XVI siècle, étude sur la psychologie de Fernel, Paris 1903.

J. Torlais, Jean Fernel, a-t-il été un grand médecin? In: Mélanges Alexandre Koyré, vol. 1, Paris 1964, S. 578-587.

${ }^{3}$ J. Fernel, De abditis rerum causis, Paris 1550 , Vorwort.

${ }^{4}$ H. Boerhaave/A. v. Haller, Methodus studii medici, Amsterdam 1751, Bd. II, S. 573.

5 E. Long, A History of Pathology, New York 1965, S. 39-41.

${ }^{6}$ Ch. Sherrington, The Endeavour of Jean Fernel, Cambridge 1946.

7 M. B. Hall, History of Science and History of Medicine. In: E. Clarke (ed.), Modern Methods in the History of Medicine, London 1971.

8 R.J. Durling, A Chronological Census of Renaissance Editions and Translations of Galen. In: Journal of the Warburg and Courtauld Institutes, Vol. XXIV, Nr. 3-4, London 1961.

${ }^{9}$ F. Valleriola, Commentarii in sex Galeni libros de morbis et symptomatibus, Lyon 1540. J. Segarra, Aitiologikae kai Pathologikae sive de morborum et symptomatum differentiis et causis, Valence 1624.

J. Riolan [d. Ä.], Annotationes in libros sex Galeni de morborum et symptomatum differentiis et causis, 1576, Manuskript in Angers.

$10 \mathrm{~J}$. Dubois (Sylvius), Methodus sex librorum Galeni in differentiis et causis morborum et symptomatum in tabellas sex, Paris 1539.

S. Dutemple, Tabulae in sex Galeni libros de morbis et symptomatibus, zitiert in Boerhaave/Haller, op. cit. sub 4, Bd. II, S. 579.

11 N. E. Nelson, Petrus Ramus and the Confusion of Logic, Rhetoric and Poetry, Ann Arbor 1947.

$12 \mathrm{~L}$. Febvre, Le problème de l'incroyance au XVI siècle (La religion de Rabelais), Paris 1942 und 1968.

${ }_{13}$ M. Schmid, Die Lehre von den Homoiomerien in der Physiologie Fernels. In: Sudhoffs Archiv 41/4 (1957), S. 317-344.

${ }^{14}$ Die Articella sind ein Sammelband mit der sog. Isagoga ad Tegni Galeni des Hunain-ibnIshak al-Ibadi (ca. 809-873), einer Einführung in die sog. Mikrotechne (Ars medica) des Galen.

15 Avicenna, Canon medicinae, Venedig 1555, Lib. I, Fen 1, doct. I, Kap.1-2; Lib. I, Fen 2, doct. I-III.

16 ibidem, Lib. I, Fen 1, doct. I, Kap. 1.

17 ibidem.

18 J. Fernel, Medicina, Paris 1554, Pars II «Pathologia», Lib. I, Kap. 1.

19 ibidem, Lib. I, Kap. 3.

20 ibidem, Lib. I, Kap. 7.

21 ibidem, Lib. I, Kap. 8. 
22 ibidem, Lib. I, Kap. 9.

23 ibidem, Lib. I, Kap. 10.

24 ibidem, Lib. I, Kap. 4.

25 ibidem, Lib. I, Kap. 6.

26 ibidem, Lib. I, Kap. 11.

27 ibidem

28 ibidem, Lib. II, Kap. 2-3.

29 ibidem, Lib. II, Kap. 7.

${ }^{30}$ O. Temkin, The History of Classification in the Medical Sciences. In: The Role and Methodology of Classification in Psychiatry and Psychopathology, Washington D. C. 1968.

31 J. Fernel, op. cit. sub 19, Pars II, Lib. I, Kap. 1.

${ }^{32}$ F. Platter, Praxeos seu de cognoscendis, praedicendis, praecavendis, curandisque affectibus homini incommodantibus tractatus primus, Basel 1602, Vorwort «Ad lectorem».

33 J. Jonston, Idea universae medicinae practicae, Amsterdam 1644, Lib. I, tit. 1-4.

${ }^{34}$ J. Fernel, op. cit. sub 19, Pars II, Lib. I, Kap. 4.

35 Ch. Sherrington, op. cit. sub 6, S. 101.

${ }^{36}$ J. Fernel, op. cit. sub 19, Pars II, Lib. I, Kap. 3.

37 ibidem, Lib. II, Kap. 2.

38 ibidem, Lib. II, Kap. 1.

39 ibidem

40 R. Virchow, Morgagni und der anatomische Gedanke, Berlin 1894, 2. Aufl.

41 A. Stettler, Zur Geschichte des Wortes «Nosologie». In: Gesnerus 33 (1976), S. 136.

${ }^{42}$ F. Platter, op. cit. sub 32, tractatus secundus, Basel 1603. Epistola dedicatoria, S. 1.

${ }^{43}$ F. Platter, Praxeos ..., 3 Bände. 1. De functionum laesionibus, Basel 1602. 2. De doloribus, Basel 1603. 3. De vitiis, Basel 1608.

44 Th. Zwinger I, In artem medicinalem Galeni tabulae et commentarii, Basel 1561.

J. J. Wecker, Medicae syntaxes, medicinam universam ordine pulcherrimo complectens, ex selectioribus medicis, tam graecis quam latinis et arabibus collectae et concinnatae, Basel 1562.

${ }^{45} \mathrm{Ph}$. Pinel, in: Dictionnaire des Sciences médicales, Paris $1812 \mathrm{ff}$. Stichwort: Nosologie.

46 F. Boissier de Sauvages, Nosologia methodica, Amsterdam 1767.

47 F. Platter, op. cit. sub 32, tractatus primus, Vorwort «Ad lectorem».

48 F. Platter, op. cit. sub 32, tractatus secundus; «Epistola dedicatoria».

49 F. Platter, De febribus, Frankfurt/Main 1597; «Epistola dedicatoria ».

50 A. Burckhardt, Geschichte der medizinischen Fakultät der Universität Basel (1460-1900), Basel 1917, S. $78 \mathrm{f}$.

${ }^{51}$ z. B. P. Roth, Theses de dolore, Basel 1583 (unter dem Vorsitz von Theodor Zwinger I gehaltene Dissertation).

${ }^{52}$ J. Fernel, op. cit. sub 19, Pars II, Lib. II, Kap. 1.

${ }^{53}$ R. Morton, Phthisiologia, in: Opera medica, Bd. I, Amsterdam 1699.

R. Morton, Pyretologia: seu exercitationes de morbis universalibus acutis, London 1692.

54 F. H. Garrison, An Introduction to the History of Medicine, Philadelphia und London 1968 (Neudruck), S. 197.

55 J. R. Partington, History of Chemistry, Bd. II, London 1961, S. $271 \mathrm{ff}$.

56 A. Hirsch, Biographisches Lexikon der hervorragenden Ärzte ..., Berlin $1929 \mathrm{ff} ., 2$. Aufl. Stichwort: Sennert. 
57 D. Sennert, Institutiones medicinae, vorgetragen seit 1603, veröffentlicht Wittenberg 1611.

D. Sennert, Medicina practica, 6 Bände, Wittenberg 1628-1635.

D. Sennert, Paralipomena, einschließlich «Vita Danielis Sennerti», Wittenberg 1642.

58 D. Sennert, Medicina practica, Lib. VI, Wittenberg 1635, Kap. 5-8.

59 D. Sennert, Paralipomena, Wittenberg 1642, Kap. «Methodus discendi medicinae».

${ }^{60}$ J. Jonston, op. cit. sub 33, Vorwort.

$61 \mathrm{Ph}$. Pinel, op. cit. sub 45.

${ }^{62}$ F. de le Boë Sylvius, Praxeos medicae idea nova, Leiden 1671. Operis distributio, § 1.

63 ibidem, Operis distributio, §3-4.

${ }^{64}$ Th. Sydenham, The Works of Thomas Sydenham, ed. Greenhill, Vol. I, London 1848, S. $13 \mathrm{ff}$.

${ }^{65}$ F. Boissier de Sauvages, op. cit. sub 46, 1. Klasse.

${ }_{66}$ W. Karst, Zur Geschichte der «Natürlichen Krankheitssysteme». Abhandlungen zur Geschichte der Medizin und der Naturwissenschaften, Heft 37, Berlin 1941, 75 S.

${ }^{67}$ F. Boissier de Sauvages, op. cit. sub 46, Kap. «Clavis classium».

\section{Summary}

For theoretical as well as for practical medical purposes "disease" forms an entity, composed of the four poles disease, symptom, cause of the disease and classification of the disease. The relationship of these four poles amongst each other forms the structure of any pathological theory. Jean Fernel (1506-1558) restructured the two poles of disease (in the anatomical substratum, the disease is to be found) and of the cause (only the causa efficiens matters for medical purpose). He introduced causality into medical thinking. Felix Platter (1536-1614) modernised the two other poles, viz. the symptom (being the perceptible) and the classification of disease conceived as syndrome. Insofar these two physicians of the Renaissance founded the structure of modern pathological theory. A short survey of the further development of the pathological theory in the 17th and 18th century is given, covering Daniel Sennert, Johannes Jonston, François de le Boë Sylvius, Thomas Sydenham and François Boissier de Sauvages.

Frau Dr. med. Antoinette Stettler

Hallwylstraße 24

3005 Bern 